\title{
Scanning Precession Electron Diffraction Study of Hybrid Precipitates in a 6xxx Series Aluminium Alloy
}

Jonas K. Sunde ${ }^{1}$, Duncan N. Johnstone ${ }^{2}$, Calin D. Marioara ${ }^{3}$, Antonius T.J. van Helvoort ${ }^{1}$, Paul A. Midgley $^{2}$, Randi Holmestad ${ }^{1}$

1. Department of Physics, Norwegian University of Science and Technology (NTNU), 7491 Trondheim, Norway

2. Department of Materials Science and Metallurgy, University of Cambridge, 27 Charles Babbage Road, Cambridge, CB3 OFS, UK

3. SINTEF Materials and Chemistry, 7465 Trondheim, Norway

Obtaining reliable and statistical assessment of nanoscale precipitates to link the microstructure with material properties is a primary objective of transmission electron microscopy (TEM) studies of agehardenable aluminium (Al) alloys. Here, it is demonstrated that nanometer-resolution scanning precession electron diffraction (SPED) [1] combined with versatile post-facto computational analysis provides a route to extract improved precipitate statistics in Al alloys. The approach developed is automated, and offers more objective assessment compared to conventional imaging procedures which require manual identification, counting and measurements. The material studied here is a common $6 \mathrm{xxx}$ series industrial alloy $(6082, \mathrm{Al}-0.72 \mathrm{Mg}-0.88 \mathrm{Si}-0.03 \mathrm{Cu}(\mathrm{wt} \%)$ ) and the methods developed are generic and applicable to any material containing nanoscale precipitates.

6xxx series $\mathrm{Al}$ alloys are $\mathrm{Mg}$ and $\mathrm{Si}$ containing age-hardenable alloys, which are strengthened by numerous metastable phases formed during heat treatment. The precipitates are needle-shaped and extend along the $<100>\mathrm{Al}$ directions. The main evolution in precipitate crystallography occurs on ageing beyond the peak hardness condition. This over-aged state is technologically important in understanding the thermal stability of $\mathrm{Al}$ alloys in applications such as heat exchangers and electrical wires. As the material is over-aged, the precipitate sizes increase and normally follows the sequence [2] $\beta$ ' $\rightarrow \beta^{\prime}, \mathrm{U} 1, \mathrm{U} 2, \mathrm{~B}$ ' $\rightarrow \beta, \mathrm{Si}$, where the different crystalline phases in 6xxx alloys are given. With $\mathrm{Cu}$ additions, the sequence changes to [3] $\beta^{\prime \prime}+\mathrm{L}+\mathrm{S}+\mathrm{QC} \rightarrow \beta^{\prime}+\mathrm{Q}^{\prime} \rightarrow \mathrm{Q}$. Different crystal structures often coexist within individual precipitate cross-sections making hybrid precipitates (e.g. Figure 1 (b)).

SPED experiments were performed using a NanoMEGAS ASTAR system fitted to a JEOL 2100F (S)TEM operated at $200 \mathrm{kV}$, with a precession angle of $0.5^{\circ}$ and a step size of $0.64-1.92 \mathrm{~nm}$. A precession electron diffraction (PED) pattern is recorded at each probe position typically yielding 160 000 diffraction patterns (DPs) covering areas of $\sim 1 \mathrm{x} 1 \mu \mathrm{m}^{2}$. The 4D-SPED dataset was then analyzed post-facto, primarily using the HyperSpy [4] Python library. Virtual dark-field (VDF) images formed by plotting the intensity within a selected sub-set of diffracting pixels, as a function of probe position, provides initial visualization of the precipitates present (Figure 1 (a)) from which the precipitate dimensions may be assessed. Depending on the material's thermo-mechanical history, a $1 \mathrm{x} 1 \mu \mathrm{m}^{2} \mathrm{scan}$ contains some 50-1000 precipitate cross-sections, which is the basis for improved statistics as compared to conventional TEM analysis.

Unsupervised learning approaches based on principal component analysis (PCA) and non-negative matrix factorization (NMF) were applied in order to find the underlying 'component patterns' that make up the data, and their associated 'loadings' at each real space pixel. The loading maps (e.g. Figure 1 (b) 
insert) indicate regions where the component patterns are significant and resemble simplified dark-field images. In the case of mixed DPs in single pixels (e.g. Figure 1 (c)) one obtains loading maps with overlapping regions of intensity where the DPs have been separated into individual components. The obtained components were compared to simulated DPs from the different known precipitate structures in order to identify and link to the real space positions of each phase. This was found to reveal phases present within single hybrid precipitate cross-sections. Decomposition reliability was assessed by correlating the results with atomic resolution images acquired from some of the very same precipitates using a double corrected JEOL ARM200CF and was found to give good agreement.

The approach developed is an important step in increasing the reliability of precipitate statistics as larger and more representative volumes can be analysed, providing powerful data to ageing models. Furthermore, because the method applies more objective data processing, the errors in acquired statistics are more readily quantifiable, and the technique provides qualitative information on the crystallography within each individual precipitate cross-section from a large number of precipitates for the first time [5].

\section{References:}

[1] JS Barnard et al, Ultramicroscopy, 174 (2017) p. 79.

[2] C Ravi and C Wolverton, Acta Materialia, 52 (2004) p. 4213.

[3] CD Marioara et al, Philosophical Magazine, 87 (2007) p. 3385.

[4] F de la Peña et al, HyperSpy - 1.1.2 (2017).

[5] JKS, CDM and RH acknowledge funding from the Research Council of Norway (247783 AMPERE) and cooperating industrial partners. DNJ acknowledges funding from the University of Cambridge and the EPSRC NanoDTC Cambridge EP/L015978/1. PAM acknowledges funding from the ERC 2915223DIMAGE and the European Commission 312483-ESTEEM2. The (S)TEM work was carried out on the NORTEM infrastructure (NFR 197405) at the TEM Gemini Centre, Trondheim, Norway.
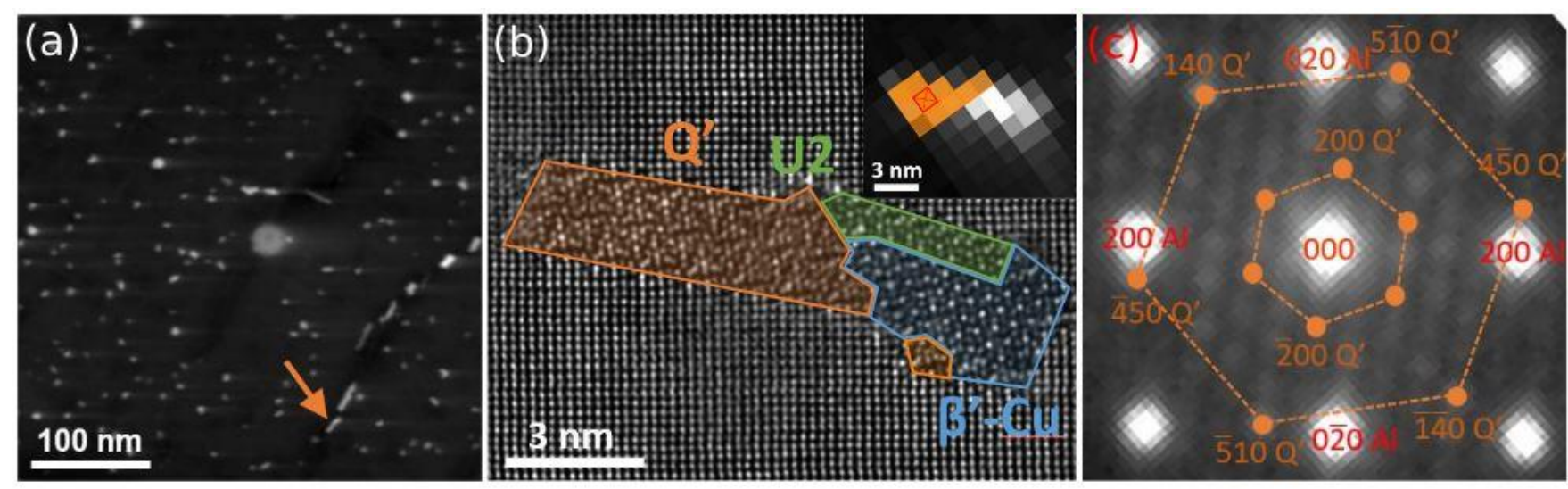

Figure 1. (a) VDF image formed from a SPED dataset acquired near to a [001]Al zone axis showing numerous precipitates. (b) High-angle annular dark-field STEM image of the precipitate highlighted in (a) reveals a hybrid Q'/U2/ $\beta$ '-Cu structure. The insert shows a cropped loading map from the same hybrid precipitate. Superposed orange coloured pixels are obtained from a loading map associated to a Q'-like factor in the decomposition data. Colour transparency qualitatively indicates phase presence in each pixel. (c) Single pixel PED pattern from the cross-marked pixel in the insert of (b). The overlaid hexagons are obtained from a simulated (kinematical) DP of a Q' structure embedded in an [001]Al matrix which showed good agreement. 